\title{
Bare Platinum Nanoparticles Deposited on Glassy Carbon Electrodes for Electrocatalytic Detection of Hydrogen Peroxide
}

\author{
Elisabetta Mazzotta ${ }^{1,}{ }^{*}$, Tiziano Di Giulio ${ }^{1}$, Valentina Mastronardi², Pier Paolo Pompa ${ }^{2}$, Mauro \\ Moglianetti $^{2, *}$, Cosimino Malitesta ${ }^{1}$ \\ ${ }^{1}$ Laboratorio di Chimica Analitica, Dipartimento di Scienze e Tecnologie Biologiche e Ambientali \\ (Di.S.Te.B.A.), Università del Salento, Lecce (Italy) \\ ${ }^{2}$ Istituto Italiano di Tecnologia IIT, Genova (Italy) \\ Corresponding Authors: Elisabetta Mazzotta (elisabetta.mazzotta@unisalento.it), Mauro \\ Moglianetti (mauro.moglianetti@iit.it)
}




\section{Experimental}

\section{Quartz Crystal Microbalance (QCM) experiments}

QCM measurements were performed using a KSV-Z500 microbalance with dissipation monitoring (KSV, Finland). A $5 \mathrm{MHz} \mathrm{SiO}$-coated quartz crystal (Biolin, Sweden) was used. The sensor was pretreated in a UV/Ozone chamber for 10 minutes before usage. The measurement was performed at $22{ }^{\circ} \mathrm{C}$ in air. The resonant frequency of the sensor was monitored for $600 \mathrm{~s}$ to acquire a steady baseline. Then, $45 \mu \mathrm{L}$ of 1:5 diluted solution of $4 \mathrm{~nm}$ PtNPs were drop-casted on the sensor surface and let to dry. Salt traces where removed rinsing with ultrapure water and solvent traces were removed under low vacuum. The sensor was then placed again in the QCM measuring chamber and the resonant frequency was recorded for $400 \mathrm{~s}$ (Fig. S3). After a rinsing step in ultrapure water followed by drying step, the sensor was placed back in the chamber.

\section{NPs Coating process with PVP}

A batch of $4 \mathrm{~nm}$ diameter citrate-capped nanoparticles have been incubated in $50 \mathrm{ppm}$ 40K PVP solution overnight under moderate stirring. The material was then used without further purification and deposited on the electrode. Electrodes tested in CV and MPAD experiments (Fig. S7) have been prepared by directly depositing $14 \mu \mathrm{L}$ of PVP-coated PtNPs solution on the electrode for achieving a loading of $0.008 \mathrm{mg}$ PtNPs, as in the case of bare PtNPs.

\section{Dynamic Light Scattering (DLS) and Z potential measurements}

Dynamic Light Scattering and Zeta potential measurements have been performed using Malvern Zetasizer instrument. The measurements performed during the removal of citrate using a basic solution are difficult to perform as the nanomaterial undergoes a destabilization process, quickly followed by aggregation and sedimentation. The results obtained should be evaluated only as an indication of the metastable states the system is passing through. 


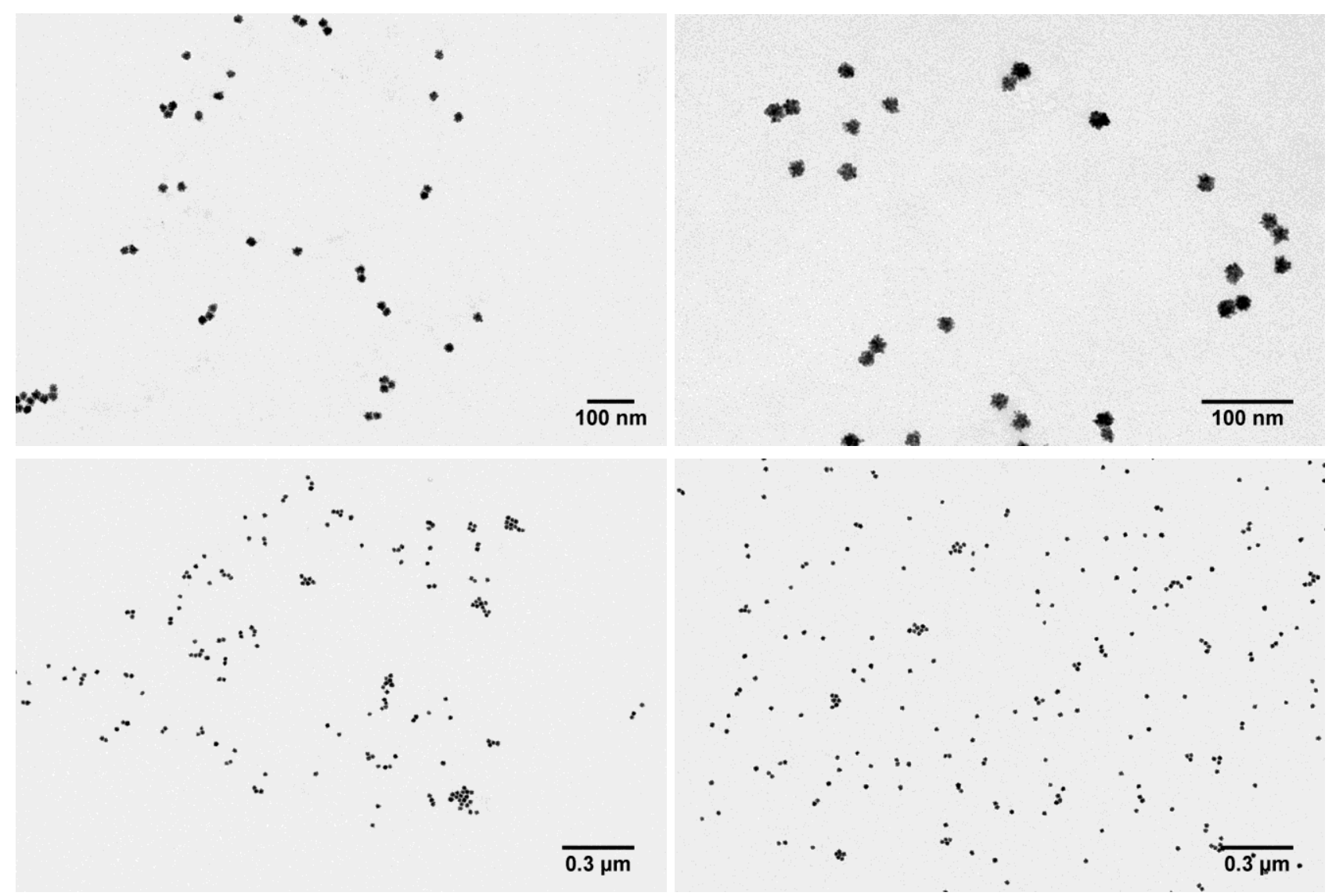

Figure S1 - Bright-field transmission electron microscopy (BF-TEM) images of the $20 \mathrm{~nm}$ spherical PtNPs at different magnifications. 

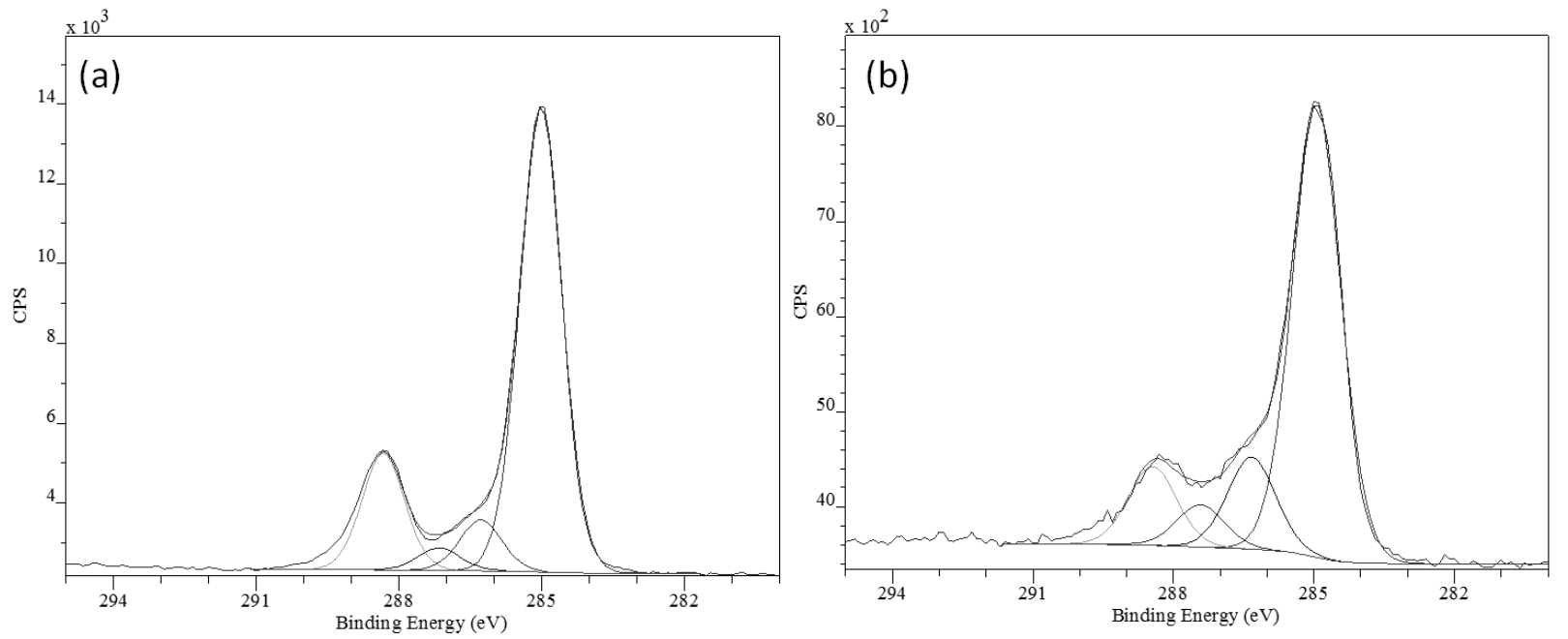

Figure S2 - XPS detailed spectra of fitted C 1s acquired on $4 \mathrm{~nm}$ PtNPs (a) stock suspension and (b) washed sample. Spectrum collected on PtNPs suspension is charging corrected. 


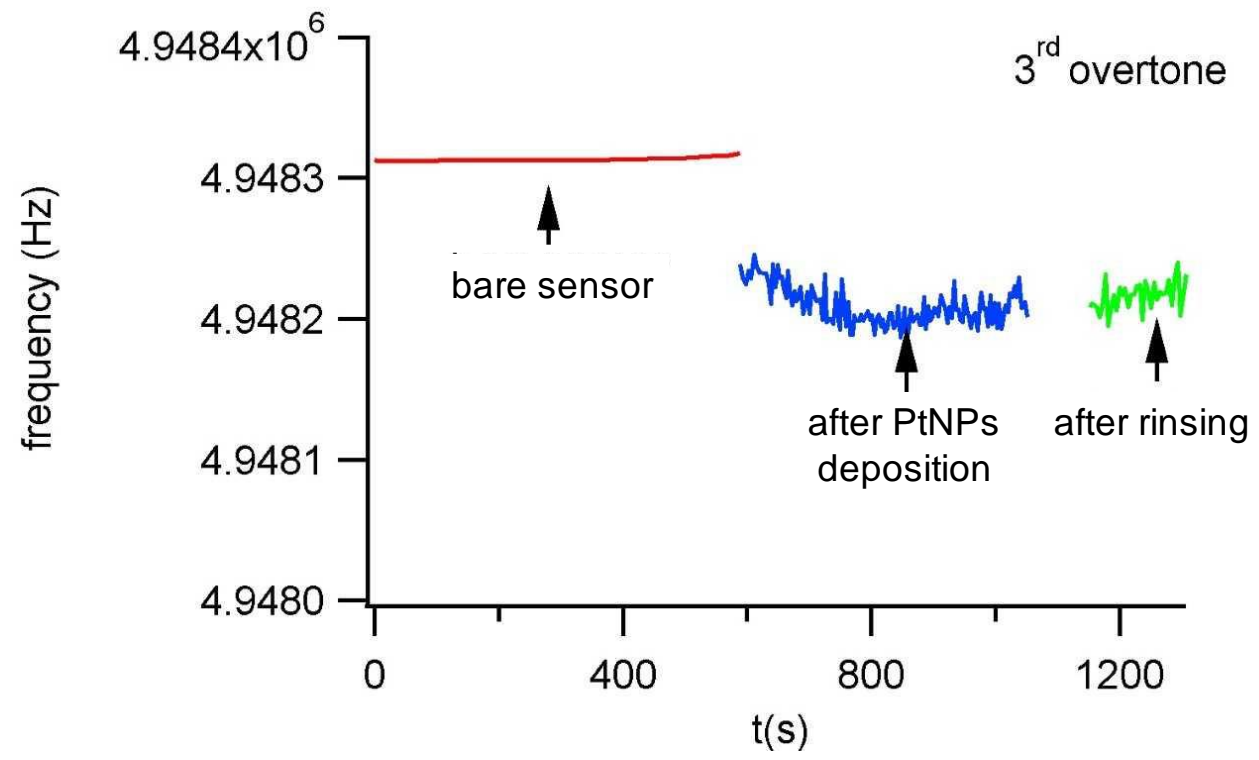

Figure S3 - QCM measurements obtained measuring the resonant frequency (3rd overtone) of a bare $\mathrm{SiO}_{2}$ quartz sensor (red curve), after drop-casting $45 \mu \mathrm{L}$ of the $4 \mathrm{~nm}$ PtNPs (blue), and after rinsing (green). 

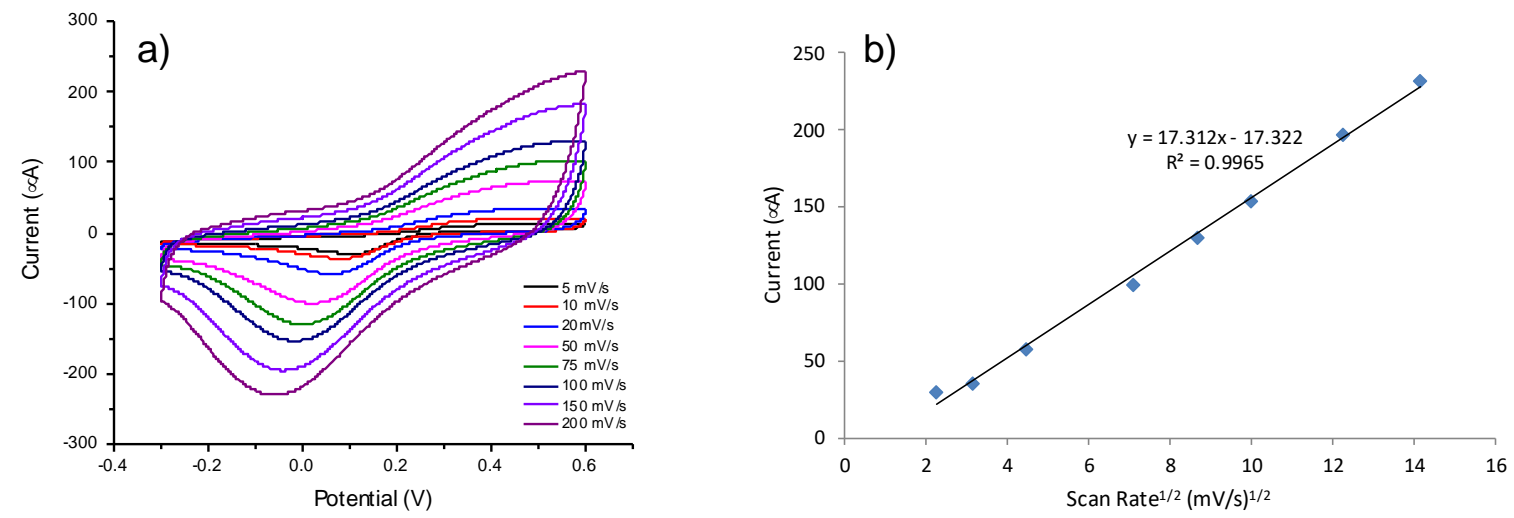

Figure S4 - a) Cyclic voltammograms on $4 \mathrm{~nm}$ PtNPs in PBS $0.1 \mathrm{M}, \mathrm{pH} 7.0$ with $\mathrm{H}_{2} \mathrm{O}_{2} 0.5 \mathrm{mM}$ at different scan rate from 5 to $200 \mathrm{mVs}^{-1}$. b) Plot of cathodic current vs square root of the scan rate from 5 to $200 \mathrm{mV} / \mathrm{s}$. 


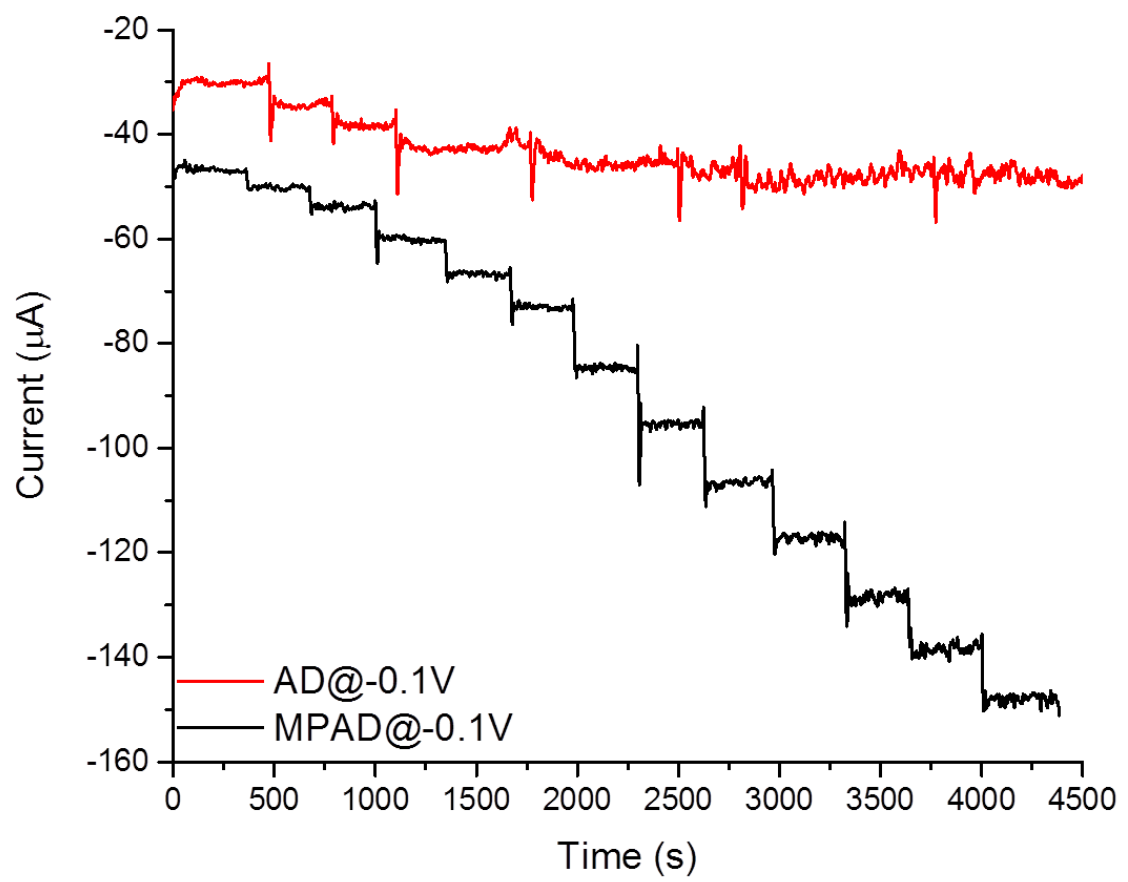

Figure S5 - Multiple Pulse Amperometric (black curve) and Amperometric (red curve) curves on $4 \mathrm{~nm}$ PtNPs in PBS 0.1 $\mathrm{M}, \mathrm{pH} 7.0$ with $\mathrm{H}_{2} \mathrm{O}_{2}$ 0.025-0.75 mM. 


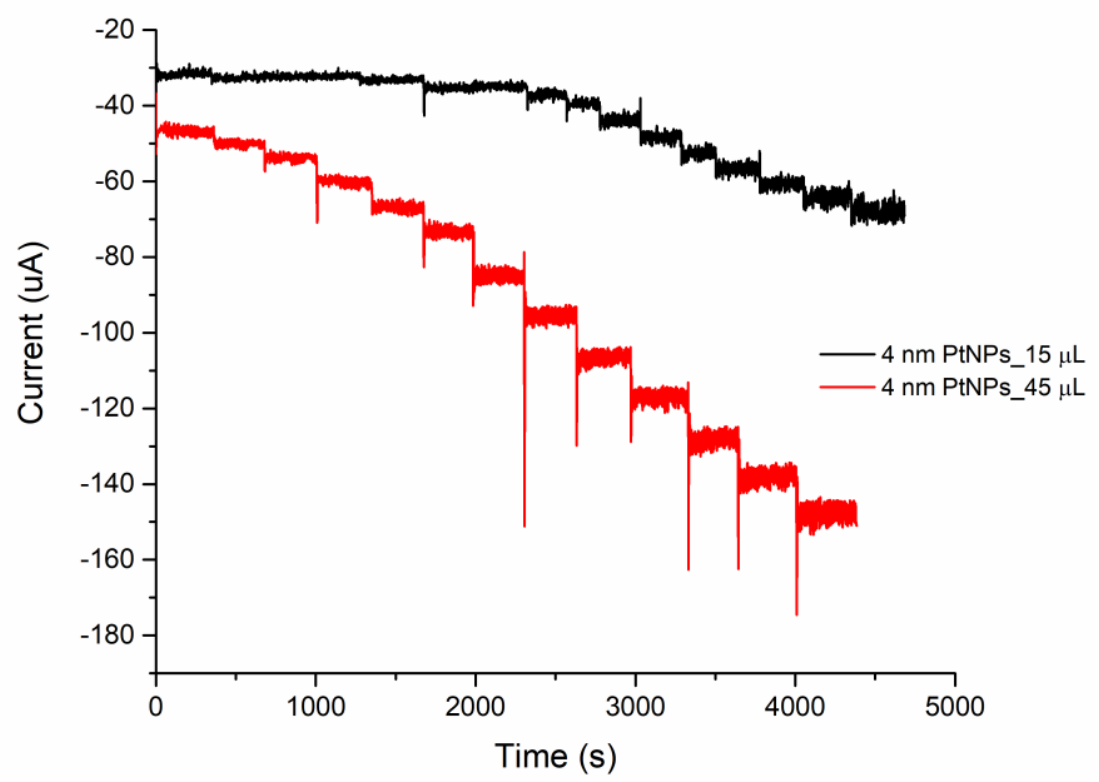

Figure S6 - Multiple Pulse Amperometric curves on electrodes prepared by drop-casting $15 \mu \mathrm{L}$ (black curve) and $45 \mu \mathrm{L}$ (red curve) of $4 \mathrm{~nm}$ PtNPs in PBS 0.1 M, pH 7.0 with $\mathrm{H}_{2} \mathrm{O}_{2}$ 0.025-0.75 mM. 
(a)

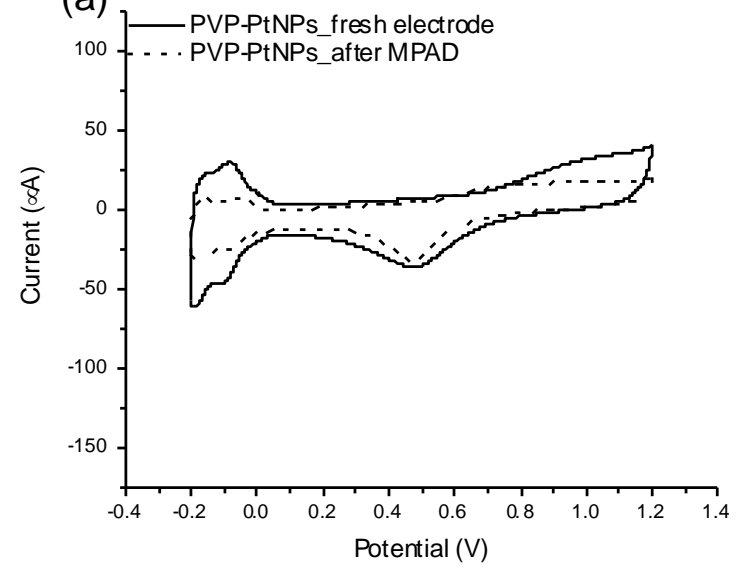

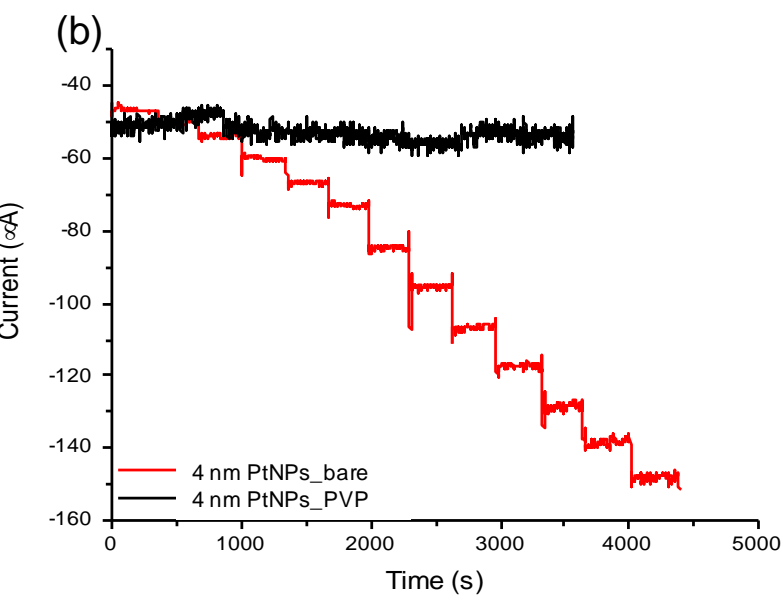

Figure S7 - (a) Cyclic voltammograms on $44 \mathrm{~nm}$ PVP-coated PtNPs in $\mathrm{H}_{2} \mathrm{SO}_{4} 0.5 \mathrm{M}$, on freshly prepared electrode (solid line) and after MPAD experiments (dotted line), scan rate $50 \mathrm{mVs}^{-1}$. Current axis scale is set as in Figure 4 to allow a rapid comparison with CVs on bare PtNPs. (b) Multiple Pulse Amperometric curves on $5 \mathrm{~nm}$ PVP-coated PtNPs (black curve) in PBS $0.1 \mathrm{M}, \mathrm{pH} 7.0$ with $\mathrm{H}_{2} \mathrm{O}_{2}$ 0.025-0.75 mM. For a comparison, also MPAD curve on bare $4 \mathrm{~nm}$ PtNPs is reported (red curve). 
Table S1 - Z potential and hydrodynamic diameter (DLS) of $20 \mathrm{~nm}$ PtNPs at different $\mathrm{pHs}$.

\begin{tabular}{|l|c|c|}
\hline Sample & Z potential (mV) & Hydrodynamic diameter (nm) \\
\hline Citrate-coated PtNPs & -31.1 & 25.5 (PDI 0.03) \\
\hline $\begin{array}{l}\text { PtNPs treated with 1M NaOH } \\
\text { aqueous solution for } 5 \text { min }\end{array}$ & -39.7 & 66.8 (PDI 0.2) \\
\hline $\begin{array}{l}\text { PtNPs treated with 1M NaOH } \\
\text { aqueous solution for } 1 \mathrm{~h}\end{array}$ & -37.5 & 110.1 (PDI 0.2) \\
\hline
\end{tabular}

